\title{
A Ioo AÑOS DE LA LEY DE INSTRUCCIÓN PRIMARIA OBLIGATORIA: ANTECEDENTES, DESARROLLO Y PROYECCIONES
}

\section{Ioo years after the Mandatory Primary Instruction Law: Background, Development and Projections}

\author{
Jaime CAiceo Escudero \\ Universidad de Santiago de Chile \\ Correo-e: jcaiceo@hotmail.com
}

Recibido: 3 de diciembre de 2020. Envío a informantes: 8 de diciembre de 2020.

Aceptación definitiva: 28 de enero de 202I

Resumen: A propósito de los ioo años de la promulgación de la Ley de Instrucción Primaria obligatoria en Chile, se persigue describir los antecedentes que condujeron a la dictación de esta ley, explicar el desarrollo legislativo de la misma y entender las proyecciones que ha tenido. Para realizar este estudio se recurrirá a un paradigma cualitativo y a la metodología histórica, revisando fuentes primarias (documentos originales) y secundarias (bibliografía existente sobre el tema). Se encontró que hubo mucha resistencia de los sectores conservadores para aprobar esta importante la ley, pero, finalmente, los argumentos de los grupos liberales primaron durante los 20 años de discusión en el Congreso y en los grupos interesados en el tema. El resultado de la promulgación de esta ley ha significado que Chile tenga cobertura prácticamente completa en el sistema escolar y tenga hoy una escolaridad promedio sobre los ıo años similar a España y Portugal.

PALABRAS ClAve: historia de la educación; escolaridad obligatoria; legislación educacional; asistencia escolar; gestión educacional.

AвSTRACT: With regard to the roo years of the promulgation of the mandatory primary instruction law in Chile, it is intended to describe the background that led to the dictation of this law, explain the legislative development of it and understand the projections it has had. To carry out this study, a qualitative paradigm 
and historical methodology will be used, reviewing primary (original documents) and secondary sources (existing literature on the subject). It was found that there was a lot of resistance from conservative sectors to approve this important law, but eventually the arguments of liberal groups prevailed during the 20 years of discussion at Congress and in groups interested in the subject. The result of the promulgation of this law has meant that Chile has practically complete coverage in the school system and today has an average schooling about to years similar to Spain and Portugal.

KEY WORDS: educational history; compulsory education; educational legislation; educational attendance; educational management.

\section{Introducción}

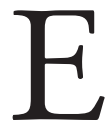
L 26 DE AGOSTO DE 1920 fue un día muy importante para la educación chilena. En efecto, se promulgó la Ley de Instrucción Primaria Obligatoria y, para celebrar tan magno acontecimiento, el inspector general de Educación Primaria, don Darío Salas, organizó tres días después un Acto Cívico en el cual desfilaron muchos estudiantes con sus maestros de las distintas escuelas de Santiago frente al Palacio de la Moneda, cantando el himno Patria Nueva, escrito por el propio Salas y compuesto por Julio Guerra; el desfile fue presidido por el presidente de la República, Juan Luis Sanfuentes, ministros de estado y otra serie de autoridades. A su vez, se decretó ese 29 de agosto como feriado nacional (Ministerio de Educación, 20I7: 8). De esta forma, finalizaba positivamente un anhelo esperado por años por los educadores que sostenían que la obligatoriedad de la educación primaria era el único mecanismo posible para que los padres enviaran a sus hijas e hijos a la escuela, a fin de poder aprender a leer e insertarse en la sociedad chilena, teniendo claridad sobre su país, su entorno social, económico y cultural y sobre sus derechos fundamentales (Ministerio de Educación, 20I7: 9).

En el presente artículo se hará una breve descripción de la educación pública en el país desde los albores de la república, a modo de antecedente; se analizará en detalle el desarrollo del proyecto de ley por cerca de 20 años para finalizar con su promulgación; finalmente, se expondrán las proyecciones que ha tenido para la educación nacional la aplicación de la mencionada ley.

\section{Metodología}

Este artículo es fruto de una investigación que se inscribe en el paradigma cualitativo, es decir, en el contexto de la epistemología de las ciencias sociales y, en este caso, con la metodología propia de los estudios históricos, recurriendo a fuentes primarias y secundarias; entre ellas, documentos originales y la vasta bibliografía existente sobre el problema en estudio; la técnica utilizada es el análisis 
documental. Para este trabajo, se asume la división que Mario Bunge realiza sobre las ciencias sociales continuando la línea de Wilhelm Dilthey, el cual realizó la importante clasificación entre las ciencias de la naturaleza y las ciencias del espíritu, precisando estas últimas como las que comprenden los fenómenos, especialmente humanos; como en este trabajo existe descripción y análisis interpretativo de hechos y documentos, está presente una episteme cercana a la fenomenología y a la hermenéutica (Caiceo, 2018a: 404-405).

\section{Resultados}

\section{I. Antecedentes}

En la denominada Patria Vieja (I8IO-I8I4) del proceso de independencia de Chile, el presidente de la Junta de Gobierno de Chile (I8II-I8I3) José Miguel Carrera crea las bases de la educación pública del futuro Estado chileno al fundar el Instituto Nacional, con base en la Academia de San Luis -fundada por don Manuel de Salas en I797-, el Convictorio Carolino -fundado en 1772 con el nombre de Real Seminario de Nobles de San Carlos, en honor al rey de España Carlos III- ${ }^{2}$, la Universidad de San Felipe -fundada por el rey Felipe V en I747- y el Seminario Conciliar. De esta forma, el Instituto abarcó los tres niveles educacionales: primario, secundario y superior; en esta institución estudiaban religiosos y civiles; su funcionamiento estuvo detenido entre I8I4 y I8I8 hasta que se logró definitivamente la independencia (Amunátegui, I889; Feliú, I950). El Instituto Nacional «ha sido señero de la educación pública chilena en sus más de dos siglos de historia; a partir de la fundación de la Universidad de Chile, en I842, se transformó en el primer Liceo público en el país» (Caiceo, 20I8b: I4).

Por otra parte, durante el gobierno de Bernardo O’Higgins (director supremo entre I8I8 y I823) se enfatizó la educación primaria e instruyó «que en cada Parroquia se fundara una escuela; para entender este hecho hay que tener presente que no había separación Iglesia Estado y, por lo tanto, los párrocos eran funcionarios públicos» (Caiceo, 20Ioa: 269); estas escuelas eran financiadas por el Estado. También se preocupó del método de enseñanza e introdujo el sistema lancasteriano o de ayuda mutua en las escuelas, consistente en que los alumnos más aventajados ayuden a sus compañeros, apoyando, de esta manera, al profesor (Mancilla, 2005); quiso formar maestros en el mismo sistema, pero ello no prosperó. A su vez, es relevante que en la Constitución de i822 se le dedique el Título VII, Capítulo Único: De la educación pública, reforzando este tipo de educación para la naciente república; en el art. 230 se establece: «La educación pública será uniforme en todas las escuelas, y se le dará toda la extensión posible en los ramos del saber,

Famoso físico, filósofo y epistemólogo argentino muerto recientemente en Canadá a los Ioo años: 24 de febrero de 2020 .

2 En este establecimiento hará sus estudios iniciales el propio general José Miguel Carrera. 
según lo permitan las circunstancias» y en el art. 23I se agrega: «Se procurará poner escuelas públicas de primeras letras en todas las poblaciones: en las que, a más de enseñarse a la juventud los principios de la religión, leer, escribir y contar, se les instruya en los deberes del hombre en sociedad».

Otro hito importante de la historia de la educación es lo que ocurre en el decenio del presidente Manuel Bulnes (I84I-I85I) y de su ministro de Justicia, Culto e Instrucción Pública Manuel Montt. En efecto, hay tres hitos fundacionales muy relevantes para el futuro educacional del país: la fundación de la Universidad de Chile $^{3}$ (I842), cuyo primer rector fue el venezolano, avecindado en Chile, Andrés Bello; la fundación de la Primera Escuela de Preceptores ${ }^{4}$, fundada el mismo año y cuyo primer rector fue el intelectual y político Domingo Faustino Sarmientos, quien se encontraba exiliado en el país -fue la segunda en América- y la Escuela de Artes y Oficios ${ }^{6}$ fundada en I849, bajo la dirección del francés Jules Jariez. En el decenio siguiente, el presidente Manuel Montt (I85I-I86I) continúa reforzando el desarrollo de la educación primaria, al traer a las religiosas del Sagrado Corazón, a quienes les entregó la dirección y administración de la Primera Escuela de Preceptoras (1854) y el 24 de noviembre de I860 promulgó la Ley General de Educación. Con esta publicación:

[...] culminó una década de debate sobre la educación primaria en Chile. Fue un hito legislativo y político. Esta ley estableció que la escuela pública, fiscal o municipal, era gratuita y que el financiamiento sería principalmente fiscal y municipal y abarcaría no solo el sueldo de los preceptores, sino la construcción, arriendo o adquisición de los locales, los textos de estudio y la formación y fomento de bibliotecas populares. (Ministerio de Educación, 2017: 7)

Primera universidad pública del Estado de Chile.

4 Originalmente se fundó en un local de la Plaza de Armas de Santiago; tres años después se trasladó a la Quinta Normal con un internado. A inicios del siglo xx se inició la construcción de un moderno edificio en la principal avenida de la capital, la avda. Bernardo O’Higgins, para cobijar a tan importante institución, denominada ahora Escuela Normal. En igio pasó a denominarse Escuela Normal Superior José Abelardo Núñez, en honor a tan distinguido educador, quien falleció ese año. La dictadura militar eliminó las escuelas normales a comienzos de 1974, traspasando la mencionada normal a la Universidad Técnica del Estado, la cual se transformó en la Universidad de Santiago de Chile en 1981 y hoy dicta la carrera de Educación Básica, formando maestras y maestros para el sistema escolar chileno, continuando con tan importante labor iniciada hace 178 años. ¡Un gran desafío para la Universidad de Santiago de Chile!

En su segundo exilio en Chile (1840-1851) -estuvo en tres momentos distintos- publicó $E d u$ cación Popular (1849), en donde expone gran parte de su pensamiento pedagógico y su proyecto de educación pública, gratuita y laica; «misma idea que se consolidará como 'estado docente’ en Chile a partir de 1860 con la dictación de la Ley de Instrucción Primaria, a la que él colaboró» (Caiceo, 2014: 195). Fue presidente de la Argentina entre i 868 y i874, impulsando con tenacidad la educación pública.

6 Esta institución que atendía la formación de técnicos se transformó en la Universidad Técnica del Estado en 1947 y a partir de 198r pasó a ser la actual Universidad de Santiago de Chile. La segunda universidad pública más importante del país. 
En Anexo del Libro Educación Popular publicado en Chile por Sarmiento, se encuentra la propuesta realizada por el educador argentino por solicitud del entonces ministro de Instrucción, Justicia y Culto de Chile, Manuel Montt, quien le hará algunos cambios y ajustes antes de presentarlo al Congreso. De esta forma, el proyecto de Sarmiento fue la base de la Ley de Instrucción Primaria promulgada en I86o cuando Montt era el presidente de la República. Con su promulgación se consolida el concepto de 'estado docente' que ha predominado hasta ahora en el sistema escolar chileno en contraposición al de la 'libertad de enseñanza', patrocinado por grupos conservadores (Caiceo, 20roa). Esta ley tuvo por finalidad ordenar la educación primaria que se había estado desarrollando durante la primera mitad del siglo XIX (Soto, 2000: 27); tendrá carácter de ley orgánica, abarcando cuatro tópicos de interés: (i) De las escuelas y el currículum; (ii) De la renta; (iii) De los preceptores, y (iv) De la inspección. Sobre el primer punto se reconocen tres tipos de escuelas: fiscales (públicas y municipales), particulares (privadas, pagadas o de pensionistas y gratuitas) y conventuales; en cuanto al currículo, en las escuelas públicas habrá de dos niveles: elementales (asignaturas: lectura y escritura del idioma patrio, doctrina y moral cristiana, elementos de aritmética práctica y el sistema legal de pesos y medidas) y superiores (asignaturas: además de las propias del nivel elemental se dará mayor ensanche a la instrucción religiosa y se enseñarán gramática castellana, aritmética, dibujo lineal, geografía, historia de Chile e historia de la Constitución Política del Estado) (Soto, 2000: 28).

Estas escuelas formadoras de maestros y maestras primarios pasaron a llamarse con el tiempo Escuelas Normales y se extendieron a lo largo de todo Chile. Un destacado educador de la segunda mitad del siglo XIx fue José Abelardo Núñez, quien fue comisionado por el gobierno de Chile en I878 para que se trasladara a Europa y Estados Unidos -quien viajó acompañado de otro gran educador, Claudio Matte, autor del Silabario que se usó en el país para enseñar a leer por cerca de roo años-, a fin de estudiar sus sistemas educativos e implementarlos en el país; ello significó virar de la pedagogía e influencia francesa en Chile a la alemana (Caiceo, 2014). Gracias al viaje de Núñez, llegaron a Chile varios educadores alemanes ${ }^{7}$ que reforzaron las Escuelas Normales y fueron claves en la fundación del Instituto Pedagógico en $1889^{8}$, en donde se comenzaron a formar profesores secundarios.

Entre ellos cabe mencionar a B. Krüssel, Hermann H. Langer, Vicetas Krzirwan, Johannes Türkes, Johanna Gremler, Antonia Güldenpffenning, Therese Kühlein, Magdalena Schöffer, María Schöffer, Amelia Witowski y Ana Zauvadsky (Mansilla, 2018: 200-20I).

8 Se inició como institución independiente para luego pasar a depender de la Universidad de Chile. Con la ley de Educación Superior de la dictadura militar, en I981 se transformó en la Academia Superior de Ciencias Pedagógicas; en 1985 se transformó en la actual Universidad Metropolitana de Ciencias de la Educación. 


\subsection{Desarrollo}

A pesar de la ley de i86o y de los esfuerzos que realizaba el Estado construyendo escuelas y contratando profesores, la situación hacia el cambio de siglo en cuanto a alfabetización de la población chilena era bastante exigua. El Estado construyó muchas escuelas, pero, en palabras de Serrano et al. (20I2a: 337), «con la activa participación de la población»; más aún,

La escuela estuvo lejos de ser una institución impuesta desde el poder estatal hacia la sociedad. Más bien ocurrió lo contrario, y el estudio empírico demuestra que fue el resultado de una demanda social que aumenta en el siglo. Ella hizo posible que la escuela pública fuese nacional no solo en un sentido político sino también territorial. (Serrano et al., 20I2b: 337-338)

Sin embargo, a pesar de ello, el efecto de aquello en la realidad no tuvo el efecto esperado. En efecto, Enrique Mac-Iver pronuncia un encendido discurso en la sesión ordinaria del Ateneo de Santiago, el I. ${ }^{\circ}$ de agosto de 1900 , en que hace un balance de la realidad chilena sobre los 82 años de vida independiente indicando que nadie podía estar feliz porque la mayoría de la población estaba en situación de pobreza y con una educación que no llegaba a la niñez y juventud; al respecto señala: «[...] a medida que las escuelas aumentan, la población escolar disminuye» (Mac-Iver, I900: 8). Esta fuerte afirmación, desconocida para la élite, que pensaba que con los presupuestos dedicados para la educación la situación era muy positiva, se vio corroborada por el Sexto Censo General de la República, realizado el 28 de noviembre de 1907, el cual arrojó que solo el 51,6 \% de la población sabía leer y que de los niños entre 5 y is años solo el $17 \%$ asistía a la escuela (Archivo Nacional, s/f). Por lo mismo, había salas vacías y se hacía imprescindible cambiar la política pública al respecto.

Agregando más antecedentes al respecto, conviene consignar lo siguiente:

La estadística puso de manifiesto la diferencia que existía entre el crecimiento intensivo del número de escuelas, la matrícula, y la efectiva llegada de los alumnos a las aulas, evidenciando que la escuela era una práctica inexistente para la gran mayoría de la población. El resultado prueba que la expansión territorial de la escuela no fue simultánea a su extensión social, y que el proceso de escolarización no estuvo determinado en forma directa por el número de escuelas abiertas. Es decir, la llegada de la escuela y la llegada a la escuela no fueron procesos idénticos. (Ponce de León, 20IO: 47I)

En ese contexto, se entiende el Proyecto de Ley sobre Instrucción Primaria Obligatoria presentado al Congreso por el senador radical Pedro Bannen el i8 de junio de I900, según el Archivo Nacional; sin embargo, según Darío Salas, ello sucedió en sesión del Senado del in de junio de 1902 por parte del senador Bannen (Salas, 1917). Sean I8 o 20 años, lo real es que este proyecto estuvo todos esos años discutiéndose en el Parlamento, pues los sectores conservadores, ligados a la Iglesia 
católica, se oponían, argumentando que ello limitaba la libertad de las familias para elegir cuándo incorporar a sus hijos e hijas a la escuela y cuándo retirarlos. Por su parte, liberales y radicales ${ }^{9}$ defendieron la no injerencia de la institución religiosa en la educación pública y laica. El senador conservador Ventura Blanco Viel planteaba al respecto:

Esto es entrar en el terreno del socialismo, que no reconoce límites. Hacer que el niño concurra a la escuela porque la sociedad cree que va a sacar provecho en ello, es sustituir el derecho del padre por el derecho de la sociedad, es hacer del hijo de familia el hijo de la sociedad, un esclavo de la sociedad, que no otra cosa importa el sistema en que para todo haya que contar con el concurso y el permiso de la sociedad, como pretenden los autores y sostenedores del proyecto en debate. Es socialista, señor Presidente, la doctrina que sustituye el derecho, el dominio y la autoridad del Estado al derecho, el dominio y la autoridad de la familia. (Blanco en «Prólogo» de Munizaga apud Salas, 1967: 18).

En forma similar a Blanco, argumentaban los senadores Rafael Errázuriz Urmeneta y Carlos Walker Martínez. Pero aparece en escena un nuevo actor, cuya influencia será determinante en la aprobación de la Ley de Instrucción Primaria Obligatoria el 26 de agosto de 1920. Se trata de Darío Salas, nacido en Puerto Saavedra -Región de La Araucanía- el 9 de mayo de i88ı, titulado de profesor normalista en Chillán en I899, profesor de castellano y francés en el Instituto Pedagógico de la Universidad de Chile en 1905 y doctor en Pedagogía en la Universidad de Nueva York en 1907 (Salas, 2004). Este educador influenciado por la pedagogía de John Dewey -a quien conoció personalmente- regresó al país para impulsar el eje central del pensamiento del educador norteamericano: a mayor educación mayor democracia. Integrado como académico a la Universidad de Chile, apoya decididamente el Proyecto que se encontraba en el Congreso al dictar en el Salón de Honor de esa Casa de Estudios, en ıэıo, la conferencia La Educación Primaria Obligatoria; con respecto a la educación y la sociedad expresa:

La educación asegura, por último, el progreso futuro: enriquece el patrimonio mental de la raza; por un lado, aumentando el caudal de conocimientos de que ésta dispone y dándole la clave para utilizar en su provecho fuerzas no utilizadas todavía y, por otro lado, desarrollando paulatinamente en ella mejores aptitudes para el aprendizaje y señalando a su perfectibilidad límites más amplios. He ahí los efectos sociales de la educación (Salas, D. apud Salas, 2004: 105).

Pero su obra más importante fue El Problema Nacional. Bases para la Reconstrucción de Nuestro Sistema Escolar Primario (1917), dedicada «[...] a los H. Diputados profesores, en la esperanza de que un impulso patriótico los mueva a luchar por la realización del programa esbozado en estas páginas» (Salas, 1967:

9 Pertenecientes al Partido Radical -laico, librepensador y masónico- fundado por Manuel Antonio Matta y Pedro León Gallo en Copiapó el 27 de diciembre de I863. 
II). Este libro es un aporte muy importante para la educación del país, puesto que realiza un «[...] diagnóstico de la situación educacional chilena, pero, a su vez, una proposición educativa en la que sobresale la importancia de la acción social de las escuelas y la unión entre democracia y educación. En su exposición está presente el pensamiento educativo de Dewey, utilizando, además, los mismos términos de la obra del norteamericano» (Caiceo, 20I8c).

El título del texto -Problema Nacional- se debe al analfabetismo; ese es el principal problema del país a los inicios del nuevo siglo; por ello, en el segundo capítulo lo aborda como el problema del analfabetismo. Recuerda el censo de 1907, indicando que había en el país «[...] un 60 \% de iletrados y un 52,8 \% de la población mayor de cinco años» (Salas, 1967: 39); enseguida precisa «[...] entre los individuos de seis a catorce años, había en 1907 un $62,8 \%$ de analfabetos, proporción [...] mucho mayor de la que habría correspondido a la población adulta considerada por sí sola» (Salas, 1967: 37). Por lo mismo, para este educador es tan importante la aprobación de la Ley de Instrucción Primaria Obligatoria, pero, al mismo tiempo, se necesita más dinero por parte del Estado, más y mejores maestros y vencer la desidia de los padres (Salas, 1917). Además de los analfabetos, Salas se refiere también a los ineficaces, a saber, el adulto analfabeto, el semiletrado, el adolescente de educación incompleta y la mujer no preparada para la vida social; para ello «[...] propone la creación de escuelas de instrucción primaria para adultos, las clases complementarias para adolescentes, escuelas de perfeccionamiento, la extensión de las instituciones de enseñanza -especialmente la técnica en el plano agrícola, comercial e industrial-y la preparación de la mujer para su función social» (Caiceo, 20I8c: Io).

A Darío Salas le preocupaba mucho la formación del maestro primario porque «[...] la calidad y condición del profesorado constituyen, sin duda alguna, el mejor exponente y el principal factor de la eficiencia de un sistema de enseñanza. Casi podría decirse que donde el maestro falla, todo falla, y que donde el maestro está a la altura de su misión, no queda en realidad a las escuelas gran cosa que pedir» (Salas, 1967: 145). Por ello propone incentivar la formación de maestros normalistas y perfeccionar a los sin título, puesto que $2 / 3$ ejercían así el ejercicio de la docencia. Propone también mejorar la situación económica de los docentes, a fin de elevar la moral del profesorado. En el fondo, «[...] si el profesor va a inculcar valores y aptitudes en los alumnos debe él primero vivirlos» (Caiceo, 20I8c: I2) porque «[...] la contracción al trabajo, la fe en la justicia, la dignidad y el respeto de sí mismo, la confianza en el esfuerzo propio, no se adquieren por la prédica, sino, sobre todo, mediante la imitación y el ejercicio» (Salas, 1967: 149).

Es necesario entender que el problema de la obligatoriedad de la educación primaria era político; ello explica la demora y la gran discusión para aprobar la ley en cuestión. $\mathrm{Al}$ respecto, se señala:

La escuela obligatoria no era solo una ordenanza para ampliar la cobertura. Era un conflicto ideológico sobre las atribuciones del Estado en materias sociales. Por eso, el proceso de discusión es tanto o más interesante que la ley misma y la 
obligatoriedad como tal es más significativa por lo que representa que por su eficacia. Su dimensión ideológica y política es la que interesa. (Serrano et al., 2012b: 32).

Finalmente el Congreso despachó la Ley de Instrucción Primaria Obligatoria y fue publicada en el Diario Oficial n. ${ }^{\circ}$ I2.755 del 26 de agosto de 1920 con el n. ${ }^{\circ} 3.654$ (Dirección Jeneral ${ }^{10}$ de Educación Primaria, 1920). Esta ley no solo hace obligatoria la educación primaria, sino que también el Estado se hace responsable de sus costos. Como deseaba el sector liberal, la ley establece una educación laica, pública y gratuita. En su art. i señalaba que «[...] todos los niños chilenos entre los 6 y 6 años, que estudien en escuelas administradas por el Estado y las municipalidades del país, estarán obligados a cursar cuatro años de enseñanza primaria». Además, para asegurar la obligatoriedad para padres y guardadores en su art. I2, dice expresamente: «El padre o guardador sufrirá la pena de presidio en su grado mínimo, o multa de uno a treinta pesos, si en el propósito de eludir las obligaciones que impone esta ley o de limitar el periodo de cumplimiento, diese información falsa acerca de la edad de su hijo o pupilo en el momento de la matrícula». Para hacer más efectiva esta política pública se probíbe el trabajo infantil a menores de $\mathrm{I} 6$ años, se establecen escuelas temporales en lugares apartados o rurales y se crean las Juntas de Educación Municipales, para censar a todos los niños en edad escolar de su respectivo territorio y ubicarlos en establecimientos de enseñanza y supervisión de las condiciones de salud de los establecimientos, que el Estado arrendaba a particulares, para habilitarlos como escuelas (Archivo Nacional de Chile, $s / f)$.

Esta ley, sin embargo, trajo consigo otras consecuencias, fuera de la obligatoriedad:

[...] constituyó una oportunidad para debatir, reformular o reafirmar aspectos tan importantes en la educación primaria chilena como el rol del Estado, el espacio para la educación particular, el carácter confesional de la enseñanza. Asimismo, la organización del sistema, sus objetivos y orientaciones estarán contemplados en la Ley y en el futuro reglamento; con propiedad puede ser considerada como el $2 .^{\circ}$ hito más significativo en el desarrollo institucional de la educación primaria, que, a su vez, imprimió una determinada orientación al desarrollo futuro de ésta. (Egaña, 2004: 29)

A su vez, Falabella y García-Huidobro (2020) señalan:

Usando un lenguaje contemporáneo, la ley de obligatoriedad es una política educativa de inclusión social. Constituye un hito en la historia de la expansión de la cobertura educacional en Chile que posibilitó el acceso a las escuelas de niños y niñas del bajo pueblo. Su puesta en marcha fue lenta y con insuficientes recursos fiscales, y tuvo mayor impacto en las ciudades que en las zonas rurales. No obstante,

1o La palabra Jeneral está escrita en el lenguaje español de la época. 
representa un logro en la consolidación de la idea de Estado docente que perduró buena parte del siglo Xx. (p. 7)

Por su parte, el propio Darío Salas, en su calidad de inspector general de Educación Primaria, dependiente del Ministerio de Educación Pública, cargo que ejerció por una década (1918-1927), puso en práctica la Ley de Instrucción Primaria Obligatoria. Durante su gestión aumentó el número de estudiantes matriculados en las escuelas. Además, mejoró la infraestructura de los establecimientos en que se formaban los futuros educadores y educadoras. En 1927 elaboró un proyecto para reformar la Educación Primaria, el cual se concretó a partir del año siguiente.

\subsection{Proyecciones}

La Ley de Instrucción Primaria Obligatoria ha tenido importantes repercusiones en el desarrollo de la educación chilena en el contexto de la idea democratizadora de Dewey, traída al país por Darío Salas. En efecto, los 4 años de escolaridad obligatoria que estableció la mencionada ley para la educación primaria se transformaron en 6 años en la Reforma iniciada por Decreto n. ${ }^{\circ} 7.500$ del ro de diciembre de 1927 ; en su art. $16 .^{\circ}$ se señala textualmente: «La enseñanza primaria se desarrollará en un período de seis años; sin embargo la enseñanza primaria rural podrá desenvolverse en un período de cuatro años». A su vez, agrega en el art. $17 .^{\circ}$ que habrá 4 tipos de escuela: «a) Escuela rural; b) Escuela granja o de concentración; c) Escuela urbana; y d) Escuela para niños indigentes, débiles y de inferioridad orgánica, anormales y retrasados mentales ${ }^{\text {II }}$. Habrá también secciones para párvulos y escuelas complementarias vespertinas y nocturnas para analfabetos de ambos sexos [...]». En el art. $7 .^{\circ}$ se precisa que «La obligación escolar durará desde los siete años cumplidos hasta los quince años de edad». En el art I. ${ }^{\circ}$ se refuerza que «La educación es propia del Estado [...]» y en el art. 5. ${ }^{\circ}$ que «La educación se orientará hacia los diferentes tipos de producción, proporcionalmente a las necesidades del país». Se consolida así la educación pragmática impulsada por el educador norteamericano, que influyó tan profundamente en gran parte del siglo Xx en Chile.

A su vez, al fundamentar la Reforma Educacional de 1965, el ministro de Educación Pública, Juan Gómez Millas, expone en la Cámara de Diputados el is de diciembre de ese año, entre otras cosas, que la cobertura educacional de la Enseñanza Primaria no superaba el $70 \%$; la deserción escolar primaria era del $68 \%$; que más del $30 \%$ de licenciados de primer ciclo no accedían a la educación secundaria, y que el nivel educativo de la población chilena era de 4,2 años y de 2,4 años en el sector rural (Castro, 20I5). Ante ello, el Gobierno de Eduardo Frei Montalva,

" Se comienza a formalizar al interior del Ministerio de Educación Pública la educación especial surgida en el país en i852 al fundarse la primera escuela de sordomudos (CAICEO, 2OIOb). 
a través del Decreto . $^{\circ}$ 27.952, modifica el sistema educacional, transformando la educación primaria en educación básica y elevando su obligatoriedad para «[...] atender a los niños entre 7 y Is años de edad; proporcionará una Educación General Común de 9 años de duración, que en un primer período la tendrá solo de 8 años» ${ }^{12}\left(\right.$ art. $\left.3 .^{\circ}\right)$. Esta reforma persigue democratizar y tecnificar la educación, según Bellei y Pérez (20ı6). El planteamiento tecnológico está inspirado en dos discípulos de Dewey, Ralph Tyler y Benjamín Bloom. Sin embargo, el hecho más significativo de esta reforma desde el punto de vista de las ideas radica en que el pensamiento laicista que había predominado desde i86o en el sistema escolar se cambió por el humanismo cristiano en lo ideológico y se mantuvo el pensamiento pedagógico de Dewey, en la persona de sus discípulos Tyler y Bloom. Ello fue posible gracias a la cristianización de Dewey realizada por el P. Alberto Hurtado en su tesis doctoral en Lovaina en 1935: hasta entonces los católicos veían con recelo la pedagogía de Dewey; ahora, valoraban los I4 principios pedagógicos descubiertos por Hurtado, quien enfatiza la función social de la educación y que la educación es el mejor instrumento de democratización. Este mismo hecho facilitó que el Estado tuviera una mirada humanista cristiana sobre su sistema escolar y no laicista (Caiceo, 2016).

Por su parte, en 2003, siendo Ricardo Lagos Escobar presidente de la República y Sergio Bitar ministro de Educación, se procede, a través de la Ley n. ${ }^{\circ}$ I9.876, a reformar la Constitución, estableciendo la obligatoriedad y gratuidad de la educación media. De esta forma en su art. único se establece: «La educación básica y la educación media son obligatorias, debiendo el Estado financiar un sistema gratuito con tal objeto, destinado a asegurar el acceso a ellas de toda la población. En el caso de la educación media este sistema, en conformidad a la ley, se extenderá hasta cumplir los 2I años de edad».

Finalmente, en el 2013, durante el primer gobierno de Sebastián Piñera, se aprueba una nueva reforma constitucional, a través de la Ley . $^{\circ}{ }^{20.710}$, la cual establece la obligatoriedad y gratuidad del kindergarten, elevando a 13 años la obligatoriedad de la escolaridad en Chile. En su art. único se establece: «Para el Estado es obligatorio promover la educación parvularia, para lo que financiará un sistema gratuito a partir del nivel medio menor ${ }^{13}$, destinado a asegurar el acceso a éste y sus niveles superiores. El segundo nivel de transición ${ }^{\mathrm{I} 4}$ es obligatorio, siendo requisito para el ingreso a la educación básica». Sin embargo, era preciso modificar la Ley General de Educación del 2009, estableciendo que para ingresar a la educación básica es necesario haber aprobado el kindergarten; el proyecto

${ }_{12}$ Hasta la actualidad se ha mantenido solo en 8 años de duración. La enseñanza secundaria se transformó en educación media con una duración de 4 años.

${ }^{13}$ En Chile se le conoce como Pre kínder y para ingresar a él el niño o niña debe tener 4 años cumplidos al 30 de marzo del año respectivo.

${ }_{14}$ En Chile se le denomina kínder y para ingresar a él el niño o niña debe tener 5 años cumplidos al 30 de marzo del año respectivo. 
respectivo ingresó al Parlamento recién el año 2018 y aún no se aprueba; por lo tanto, aún no ha entrado en vigencia.

En el contexto anterior, hay que entender el significado de la aprobación de esta ley hace roo años, a partir de la disputa de las élites liberales y conservadoras de la época y que recién en el siglo Xxi ha cobrado relevancia, la educación como un derecho social:

Había, finalmente, un aspecto común a las miradas de la elite de la época: su visión del sujeto que debía educarse. Se construyó así, en el imaginario político, una necesidad pública, de instruir y alfabetizar a las clases populares, lo cual representaba un esperado avance en cobertura, expansión e integración escolar. Esta ley estableció de este modo, no solamente que la educación primaria fuese obligatoria, sino que sea el Estado el que defina, por sobre la decisión de las familias, que la educación es un derecho social para todas las niñas y niños del territorio chileno. (Falabella y García Huidobro, 2020: 7-8)

\section{Discusión}

Después de ioo años de la dictación de la Ley de Instrucción Primaria Obligatoria se puede constatar que ha dado sus frutos, puesto que la cobertura del sistema educativo es total en oferta para la educación básica y media, aunque aún existen problemas de deserción y abandono del sistema de alrededor del $2 \%$ en la educación básica ${ }^{15}$ y de un $5 \%$ en la educación media ${ }^{16}$. Ello ha significado que Chile tenga niveles de escolaridad de más de io años similares a países como España y Portugal, miembros de la $\mathrm{OCDE}^{17}$ y a la cual Chile se integró en el 20IO, convirtiéndose en el miembro . $^{\circ}$ 3I de este importante organismo y el primero de América del Sur. Con el avance en los años de escolaridad, se ha concretado el planteamiento democratizador de Dewey en la persona de Salas. En efecto, tal como se indicó anteriormente, el apoyo del educador chileno al proceso de aprobación de la ley de I920 con la publicación de su obra en 1917, basada en el planteamiento de su maestro Dewey, fue relevante; con su afán democratizador continuará insistiendo en que la educación para todos los chilenos y chilenas es significativa si se desea afianzar la democracia; sus anhelos y acciones se han visto coronados ioo años después.

Sin embargo, el gran problema que subsiste es la calidad de la enseñanza; desde la vuelta a la democracia se han incrementado los programas y las políticas públicas para mejorarla, pero el problema subsiste: en las pruebas PISA, Chile es

is Educación primaria en otros países.

16 Educación secundaria en otros países. Datos entregados por la Agencia de Calidad del Ministerio de Educación de Chile.

${ }^{17}$ Organización para la Cooperación y el Desarrollo Económicos. Según estadísticas al 2015, Chile tenía un promedio de escolaridad de 9,9, España 9,8 y Portugal 8,9 (DB City.com [s/f]). 
uno de los últimos países de la $\mathrm{OCDE}^{\mathrm{I} 8}$-organización a la cual pertenece desde 20Io- y a nivel de los 78 países que la rindieron en 2or8, Chile ocupa el puesto 43 en lectura, 45 en ciencia y 59 en matemática, aunque a nivel latinoamericano ocupa el primer lugar en lectura y ciencia y el segundo lugar en matemática. ¡Un gran desafío que superar para Chile y Latinoamérica! Se está bajo la media.

El problema relevante en cobertura insuficiente que persiste es en la educación parvularia, que se mantiene con un déficit cercano al $50 \%$. Producto de la pandemia, se necesitarán muchas salas cunas y jardines infantiles donde tendrán que dejar en forma segura sus hijos e hijas las madres que deberán salir a trabajar para salir del estado de pobreza en que quedará un número grande de familias; allí, los niños y las niñas no solo estarán seguros, sino que especialmente recibirán educación y alimentación. Chile, en principio, retrocederá su nivel de ingresos entre un I2 \% y un $\mathrm{I}_{5} \%$ y la pobreza subirá de un $9 \%$ a un $\mathrm{I}_{5} \%$. El esfuerzo directo del Estado será insuficiente; por lo mismo, debiera aprobarse financiamiento público para las salas cunas y jardines infantiles que mantienen apoyos privados, al igual como se financian los establecimientos particulares gratuitos desde larga data en el país. Para robustecer el rol del Estado, una de las políticas que debieran implementarse es mejorar sustantivamente la educación pública -tanto en infraestructura como en medios pedagógicos y calidad de los aprendizajes-, que hoy día cobija solo al 37 \% de los estudiantes del sistema escolar; cuando ello suceda, la matrícula de estos establecimientos subirá en desmedro de la educación particular subvencionada por el Estado, que equivale al $55 \%$ de los estudiantes; el $8 \%$ restante es particular pagada.

Es importante recordar el primer centenario de la Ley de Instrucción Primaria Obligatoria por dos razones fundamentales: (i) Dar a conocer lo difícil que fue su promulgación por el sentido político de la misma al hacer responsable al Estado para educar a su población como un derecho social y (ii) las consecuencias que ha tenido en el tiempo esta ley, al llegar Chile a los ıoo años de la promulgación de la misma a haber establecido I2 años de escolaridad obligatoria para todos los residentes en el territorio nacional frente a los 4 años originales.

\section{Bibliografía}

Amunátegui, Domingo: Los Primeros Años del Instituto Nacional, Santiago de Chile, Imprenta Cervantes, I889.

Archivo Nacional de Chile: «La Ley de Instrucción Primaria Obligatoria, 26 de agosto de I920», (s/f). Disponible en: HTTPS://Www.ARCHIVONACIONAL.GOB.CL/6I6/W3-ARTICLE-809o. HTML?_NOREDIRECT=I. Consultada el I6 de septiembre de 2020.

Bellei, Cristián y Pérez, Camila: «Democratizar y tecnificar la educación. La reforma educacional de Eduardo Frei Montalva», en Huneeus, Carlos y Couso, Javier (eds.): Eduardo

18 Organización para la Cooperación y el Desarrollo Económicos. Está compuesta en la actualidad por 37 países, 3 de los cuales son latinoamericanos. 
Frei Montalva: Un gobierno reformista. A 50 años de la «Revolución en Libertad», Santiago de Chile, Editorial Universitaria, 20I6, pp. 207-24I.

Biblioteca del Congreso Nacional: «Decreto n. ${ }^{\circ}$ 27.952: Modifica Sistema Educacional», Diario Oficial, Santiago de Chile, 20 de diciembre, 1965. Disponible en: https://legislacion-oficial.vlex.cl/vid/decreto-modifica-sistema-educacional-4976504I8. Consultado el i6 de septiembre de 2020 .

Biblioteca del Congreso Nacional: «Ley n. ${ }^{\circ}$ 19.876: Reforma Constitucional que establece la obligatoriedad y gratuidad de la educación media», Diario Oficial, Santiago de Chile, 22 de mayo, 2003. Disponible en: https://www.bcn.cl/leychile/navegar?idNorma=210495. Consultada el I6 de septiembre de 2020.

Biblioteca del Congreso Nacional: «Ley n. ${ }^{\circ}$ 20.710: Reforma Constitucional que establece la obligatoriedad del segundo nivel de transición y crea un sistema de financiamiento gratuito desde el nivel medio menor», Diario Oficial, Santiago de Chile, in de diciembre, 2013. Disponible en: https://www.bcn.cl/leychile/navegar?idNorma=I057032. Consultada el i6 de septiembre de 2020.

Caiceo, Jaime: «Presencias de la Iglesia y el Estado Chileno Republicano en el Sistema Educativo», en Carvalho, Carlos y Neto, Wenceslau (orgs.): Estado, Igreja e Educação: O Mundo Ibero-americano nos Séculos XIX e XX, Campinas, SP, Editora Alínea, 20Ioa, pp. 265-299.

Caiceo, Jaime: «Esbozo de la Educación Especial en Chile: 1850-1980», Revista Educación y Pedagogía, n. ${ }^{\circ}$ 57, vol. 22 (20Iob), pp. 31-50. Disponible en: file://C:/Users/Jaime/AppData/Local/Temp/Dialnet-EsbozoDeLaEducacionEspecialEnChiler8501980-3648667.pdf. Consultada el 16 de septiembre de 2020.

Caiceo, Jaime: «Influencia Educacional de Domingo Faustino Sarmiento en Chile», Revista Dos Puntas, año VI, n. ${ }^{\circ}$ Io (2014), pp. I83-203. Disponible en: file://C:/Users/Jaime/ AppData/Local/Temp/Dialnet-InfluenciaEducacionalDeDomingoFaustinoSarmientoEnC-5068244.pdf. o buscar en: https://dialnet.unirioja.es/revista/20347/A/20r4. Consultada el I4 de septiembre de 2020.

Caiceo, Jaime: La Pedagogía de Dewey en Chile: Su Presencia, a través de sus Discípulos, durante el siglo XX, Santiago de Chile, Ediciones Universidad de Santiago de Chile, 2016.

Caiceo, Jaime: «El porqué del desarrollo insuficiente de la filosofía de las ciencias sociales», El Futuro del Pasado, n. ${ }^{\circ}$ (2018a), pp. 393-418. DOI: http://dx.doi.org/Io.I4516/ fdp.2018.009.00I.0I4

CaiceO, Jaime: «Don Manuel de Salas y la Academia de San Luis, activos participantes del proceso emancipador chileno", Revista Teoria e Prática da Educação, v. 21, n. 3 (2018b), pp. 03-I4. DOI: https://doi.org/I0.4025/tpe.v21i3.45568

Caiceo, Jaime: «Democracia y Educación de John Dewey presente en El Problema Nacional de Darío Salas: A propósito del Centenario de ambas obras», Revista HISTEDBR On-line, vol. I8, n. ${ }^{\circ}$ I(75) (20I8c), pp. 5-I6. DOI: https://doi.org/I0.20396/rho.vi8ir.8651792

CASTRo, Cristhián: La reforma educacional de la década de los 60 en Chile, Tesis para optar al título de Profesor de Historia y Ciencias Sociales, Universidad Austral de Chile, 2015. Disponible en: http://cybertesis.uach.cl/tesis/uach/2015/ffc355r/doc/ffc355r.pdf. Consultada el I6 de septiembre de 2020.

DB City.com: Años Promedios de Escolarización, s/f. Disponible en: https://es.db-city.com/ $\mathrm{Pa}_{3} \mathrm{C}_{3} \%$ ADs--A\% $\mathrm{C}_{3} \% \mathrm{~B}_{10}$-promedio-de-escolarizaci\% $\mathrm{C}_{3} \% \mathrm{~B}_{3}$ n. Consultada el I4 $_{4}$ de abril de 202I.

Dirección Jeneral de Educación Primaria: «Lei n. ${ }^{\circ}$ 3.654", Diario Oficial n. ${ }^{\circ}$ 12.755, Santiago de Chile, 26 de agosto, I920. Disponible en: http://www.memoriachilena.gob.cl/602/w3-article-94746.html. Consultada el I6 de septiembre de 2020. 
A IOO AÑOS DE LA LEY DE INSTRUCCIÓN PRIMARIA OBLIGATORIA:

ANTECEDENTES, DESARROLLO Y PROYECCIONES

JAIME CAICEO ESCUDERO

Egaña, Loreto: «Ley de Instrucción Primaria Obligatoria: Un debate político», Revista de Educación, 315 (2004), pp. I4-29. Disponible en: http://bibliorepo.umce.cl/revista_educacion/2004/315/I4_29.pdf. Consultada el 22 de noviembre de 2020.

Falabella, Alejandra y García-Huidobro, Juan Eduardo (eds.): A roo años de la ley de instrucción primaria obligatoria: La educación chilena en el pasado, presente y futuro, Santiago de Chile, Facultad de Educación, Universidad Alberto Hurtado, 2020.

Feliú Cruz, Guillermo: La Fundación del Instituto Nacional, Santiago de Chile, Imprenta Cultura, 1950.

Mac-Iver, Enrique: Discurso sobre la Crisis moral de la República, Santiago de Chile, Imprenta Moderna, I900.

Mancilla, Arturo: Antecedentes para una historia de la educación primaria en Chile. Siglos $X I X$ y comienzos del XX, Tesis para optar al grado de Magíster en Historia, Universidad de Chile, 2005. Disponible en: http://repositorio.uchile.cl/bitstream/handle/2250/133702/Antecedentes-para-una-historia-de-la-educacion-primaria-en-Chile.pdf;sequence $=$ I. Consultada el I4 de septiembre de 2020.

Mansilla, Juan: «Influencia alemana en la reforma de las Escuelas Normales de Preceptores y Preceptoras en el centro sur de Chile, 1883-1920", Revista Historia de la Educación Latinoamericana, 20(31) (2018), pp. I89-209. DOI: https://doi.org/I0.19053/oI227238.8574

Ministerio de EdUCACión: «Ministerio de Educación: i8o años de historia, i8o años de servicio», Revista de Educación, n. ${ }^{\circ}$ 38I (2017), pp. 4-15. Disponible en: http://www.revistadeeducacion.cl/ministerio-de-educacion-I8o-anos-de-historia-I8o-anos-de-servicio/. Consultada el I4 de septiembre de 2020.

Ministerio de Educación Pública: Decreto n. ${ }^{\circ} 7.500$ sobre Reforma Educacional, Santiago de Chile, Imprenta Nacional, I928. Disponible en: http://www.memoriachilena.gob.cl/archivos2/pdfs/MCoo57582.pdf. Consultada el I6 de septiembre de 2020.

Ponce de León, MACARENA: "La llegada de la escuela y la llegada a la escuela. La extensión de la educación primaria en Chile, I840-1907", Historia, 43(2) (2010), pp. 449-486. DOI: http:// dx.doi.org/10.4067/S0717-71942010000200004

República De Chile: Constitución Política del Estado de Chile, Santiago de Chile, Imprenta del Estado, I822. Disponible en: http://www.memoriachilena.gob.cl/6o2/w3-article-8977. html. Consultada el I4 de septiembre de 2020.

SAlas, Darío: El problema nacional. Bases para la reconstrucción de nuestro sistema escolar primario, Santiago de Chile, Sociedad Imprenta y Litografía Universo, 1917.

SAlAS, Darío: El problema nacional. Bases para la reconstrucción de nuestro sistema escolar primario, 2. ${ }^{a}$ ed., Santiago de Chile, Editorial Universitaria, 1967.

SAlas, Emma: «Darío Enrique Salas Días: Un educador de excepción», Pensamiento Educativo. Revista de Investigación Educacional Latinoamericana, 34(I) (2004), pp. 99-II8. Disponible en: http://pensamientoeducativo.uc.cl/index.php/pel/article/view/280/588. Consultada el I6 de septiembre de 2020 .

Sarmiento, Domingo Faustino: De la Educación Popular, Santiago de Chile, Imprenta de Julio Belín i Compañía, I849.

Serrano, Sol; Ponce de León, Macarena y Rengifo, Francisca: Historia de la Educación en Chile (I8Io-20I0). Tomo I: Aprender a leer y escribir (I8Io-I880), Santiago de Chile, Taurus, 20I2a.

Serrano, Sol; Ponce de León, Macarena y Rengifo, Francisca (eds.): Historia de la Educación en Chile (1810-2010). Tomo II: La educación nacional (1880-1930), Santiago de Chile, Taurus, 2oI2b.

Soto, Fredy: Historia de la Educación Chilena, Santiago de Chile, Centro de Perfeccionamiento, Experimentación e Investigaciones Pedagógicas del Ministerio de Educación, 2000. 
